\title{
Conversion of a DWDM signal to a single Nyquist channel based on a complete optical
} Fourier transformation

Guan, Pengyu; Røge, Kasper Meldgaard; Mulvad, Hans Christian Hansen; Hu, Hao; Morioka, Toshio; Oxenløwe, Leif Katsuo

\section{Published in:}

Proceedings of 2014 European Conference on Optical Communication

Link to article, DOI:

10.1109/ECOC.2014.6964033

Publication date:

2014

Document Version

Peer reviewed version

Link back to DTU Orbit

Citation (APA):

Guan, P., Røge, K. M., Mulvad, H. C. H., Hu, H., Morioka, T., \& Oxenløwe, L. K. (2014). Conversion of a DWDM signal to a single Nyquist channel based on a complete optical Fourier transformation. In Proceedings of 2014 European Conference on Optical Communication (pp. 1-3). IEEE. https://doi.org/10.1109/ECOC.2014.6964033

\section{General rights}

Copyright and moral rights for the publications made accessible in the public portal are retained by the authors and/or other copyright owners and it is a condition of accessing publications that users recognise and abide by the legal requirements associated with these rights.

- Users may download and print one copy of any publication from the public portal for the purpose of private study or research.

- You may not further distribute the material or use it for any profit-making activity or commercial gain

- You may freely distribute the URL identifying the publication in the public portal 


\title{
Conversion of a DWDM Signal to a Single Nyquist Channel based on a Complete Optical Fourier Transformation
}

\author{
P. Guan, K. M. Røge, H. C. Hansen Mulvad, H. Hu, T. Morioka, L. K. Oxenløwe
}

DTU Fotonik, Department of Photonics Engineering, Technical University of Denmark, Ørsteds Plads, Building 343, DK-2800 Kgs. Lyngby, Denmark, pengu@fotonik.dtu.dk

\begin{abstract}
We propose a DWDM-to-Nyquist channel conversion scheme based on complete Optical Fourier Transformation and optical Nyquist filtering. We demonstrate conversion from 50-GHz-grid $16 \times 10$ Gbit/s DPSK DWDM to a 160-Gbit/s Nyquist channel (0.9 symbol/s/Hz spectral efficiency) with $1.4 \mathrm{~dB}$ power penalty.
\end{abstract}

\section{Introduction}

In the past decades, dense wavelength division multiplexing (DWDM) has enabled significant increases in capacity, but this is no longer sufficient. Due to the rapid increase in Internet traffic, it is soon expected to reach the capacity of the currently installed communication systems ${ }^{1}$. Recently, a Nyquist pulse technique has been proposed, which allows the signal bandwidth to be narrowed without intersymbol interference (ISI), enabling terabit/s transmission with high spectral efficiency ${ }^{2}$. Over the last ten years, the time-domain optical Fourier transformation (OFT) or time lens principle has been shown to be a very versatile tool for ultrafast signal processing. It can be used for manipulating the shapes of optical signals in both the time- and frequency-domains. This has led to demonstrations of e.g. distortion-less transmission $^{3}$, OTDM-to-WDM conversion ${ }^{4}$ and all-optical OFDM transmission systems ${ }^{5}$.

In this paper, we propose a DWDM to Nyquist channel conversion scheme based on complete OFT and narrow optical Nyquist filtering. Using this scheme, we convert 16 10-Gbit/s differential phase-shift keying (DPSK) channels placed on a $50-\mathrm{GHz}$ DWDM grid to a 160-Gbit/s Nyquist channel with a total bandwidth of $178 \mathrm{GHz}$, thus greatly improving the spectral efficiency from 0.2 to $0.9 \mathrm{symbol} / \mathrm{s} / \mathrm{Hz}$. A full bit error rate (BER) characterization is performed, revealing a converted Nyquist channel with $\mathrm{BER}<10^{-9}$ and only $1.4 \mathrm{~dB}$ average power penalty compared to the DWDM system. This technique could provide a simple approach to enhance the spectral efficiency of existing commercial DWDM-based systems without large system upgrades, yielding larger total system capacity.

\section{Principle}

The complete OFT is based on two quadratic phase-modulation stages (time lenses) with chirp rate $K$, separated by a dispersion medium of $D=\beta_{2} L$ (where $\beta_{2}$ is 2 nd order dispersion and $L$ is length), that satisfies $K=1 / D$ (a $K-D-K$ configuration). Unlike other partial OFT schemes $^{3,4}$, this configuration enables both timeto-frequency and frequency-to-time conversion at the same time, thus performing an exchange between the temporal and spectral envelopes of the input signal. The chirp rate $K$ determines the scaling factor between the time and frequency domains according to $\Delta t=\Delta \omega / K$.

The principle of the DWDM to Nyquist channel conversion scheme is shown in Fig. 1. The DWDM transmitter generates an $\mathrm{N}$-channel DWDM signal with frequency spacing $\Delta \omega$ at symbol rate $f_{\mathrm{S}}(\mathbf{A})$. The DWDM channels with flat-top temporal waveforms are bit-wise synchronized. A complete OFT using a $K-D-K$ configuration is then applied to exchange the temporal and spectral envelopes of the input DWDM signal. After the OFT, the N-channel DWDM signal (A) is thus converted to an OTDM signal with channel spacing $\Delta T=\Delta \omega / K$ and overlapping flat-top channel spectra. (B). A rectangular optical bandpass filter with bandwidth $\mathrm{BW}=2 \pi / \Delta T$ is then used to Nyquist filter the center of the overlapping spectra, resulting in sinc-shaped Nyquist pulses with a half zero-crossing pulse duration given by $2 \pi / B W$, set equal to $\Delta T$ to achieve orthogonality in the time-domain (C). To achieve maximum spectral efficiency, the OTDM channel spacing $\Delta T$ is set equal to $1 /\left(N f_{\mathrm{S}}\right)$. The Nyquist OTDM channel can be received in the time-domain

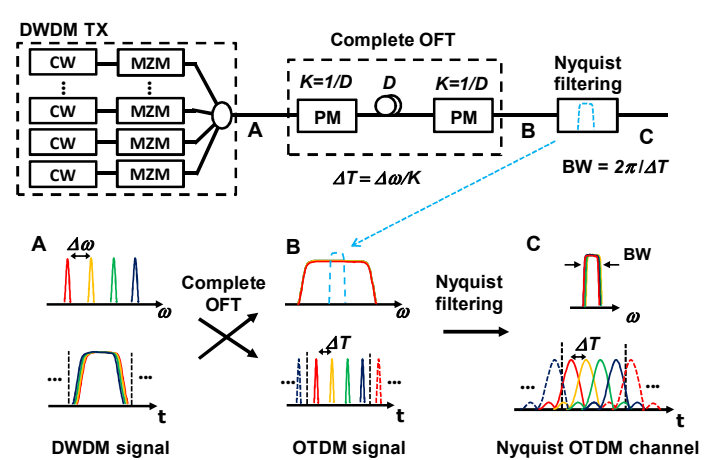

Fig. 1: Principle of DWDM signal to a single Nyquist channel conversion. 


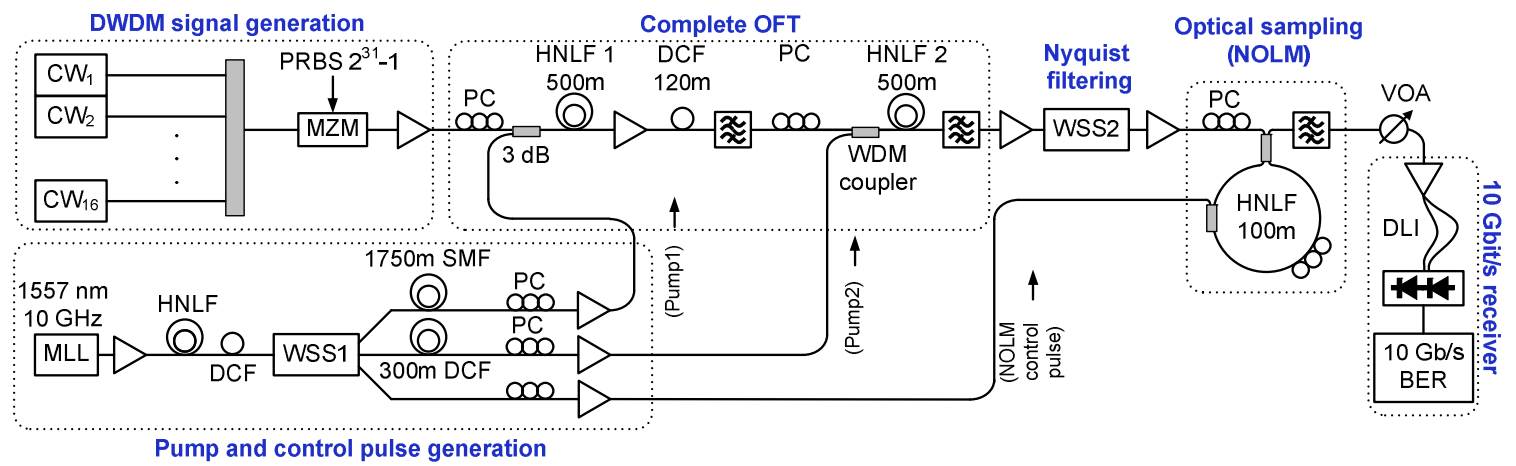

Fig. 2: Experimental setup of $16 \times 10 \mathrm{Gbit/s}$ DPSK DWDM to a single Nyquist channel conversion

using optical sampling ${ }^{2}$ or in the frequencydomain using OFT-based time-to-frequency conversion ${ }^{6}$.

\section{Experimental setup and results}

The experimental setup is shown in Fig. 1. The DWDM signal source consists of 16 distributed feedback laser diodes (DFB-LDs), centered from 1547.40 to $1553.32 \mathrm{~nm}$ with a $50 \mathrm{GHz}(0.4 \mathrm{~nm})$ spacing. The outputs of the $\mathrm{CW}$ lasers are then DPSK modulated with a $10 \mathrm{Gbit} / \mathrm{s} 2^{31}-1$ PRBS in a Mach-Zehnder modulator, resulting in a $16 \times 10$ Gbit/s DPSK DWDM signal placed on a $50 \mathrm{GHz}$ grid as shown in Fig.3 (a). Then, a complete OFT is used for the DWDM to Nyquist channel conversion. The quadratic phase modulation is implemented based on a four wave mixing (FWM) process in a highly nonlinear fiber (HNLF) using linearly chirped rectangular pump pulses. As a FWM pump pulse source, we use a mode-locked laser at $1557 \mathrm{~nm}$, and its spectrum is broadened via self-phase modulation in a 400-m dispersion-flattened HNLF (DF-HNLF) with normal dispersion. To obtain linearly chirped pumps, the output spectrum of the DFHNLF is filtered using a wavelength selective switch (WSS ${ }_{1}$ ), and each pump is subsequently propagated through an appropriate length of dispersion medium. The first pump is dispersed in a $1750 \mathrm{~m} \mathrm{SMF}$ in order to convert a $50 \mathrm{GHz}$ frequency grid to $6 \mathrm{ps}$ temporal spacing (scaling factor $\left.\Delta t / \Delta \omega=19.1 \mathrm{ps}^{2}\right)$, resulting in a $55 \mathrm{ps}$ chirped rectangular pump pulse. As the data signal is phase-conjugated after the first FWM process, the second pump is dispersed in a 300 $\mathrm{m}$ dispersion compensating fiber (DCF) having the opposite dispersion value of the $1750 \mathrm{~m}$ SMF, in order to achieve the same chirp rate K. The central wavelengths of the pumps are set at $1563 \mathrm{~nm}$.

The first FWM output is shown in Fig. 3 (a). After extraction, the idler is dispersed in $120 \mathrm{~m} \mathrm{DCF}$, then combined with pump $_{2}$ and coupled into $\mathrm{HNLF}_{2}$ for the second FWM process. The resulting spectrum is shown in Fig. 3(b). The generated idler is the $160 \mathrm{Gbit} / \mathrm{s}$ OTDM signal converted from the 16-channel DWDM signal. Note that the 55-ps rectangular RZ waveform obtained after the first OFT is converted to a 3.7 $\mathrm{nm}$ rectangular spectrum according to the scaling factor of the complete OFT. Fig. 3(c) shows the resulting OTDM waveform with 6 ps spacing, and a short guard interval between every 16 tributaries. A $166 \mathrm{GHz}$ rectangular Nyquist filter implemented by $\mathrm{WSS}_{2}$ is then used to filter the central part of the OTDM spectrum into a Nyquist OTDM channel as shown in Fig. 3(b). The resulting Nyquist OTDM spectrum is shown in Fig. 4(a). A $167 \mathrm{GHz}$ rectangular spectrum is achieved (measured at the $6 \mathrm{~dB}$ level). However, due to the limited frequency resolution of the WSS, the width measured at

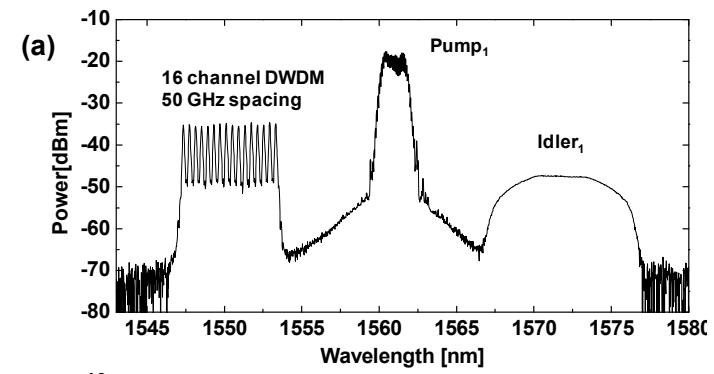

(b)

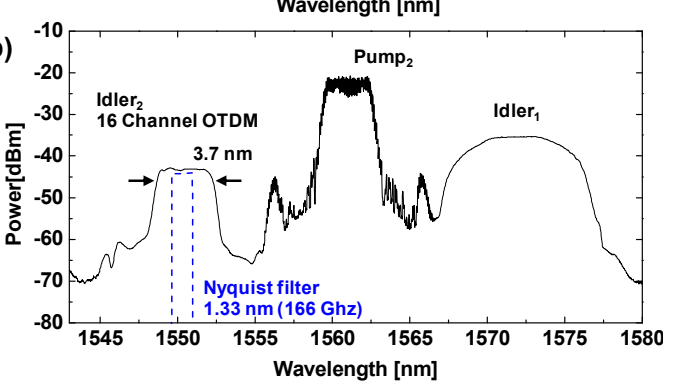

(c)

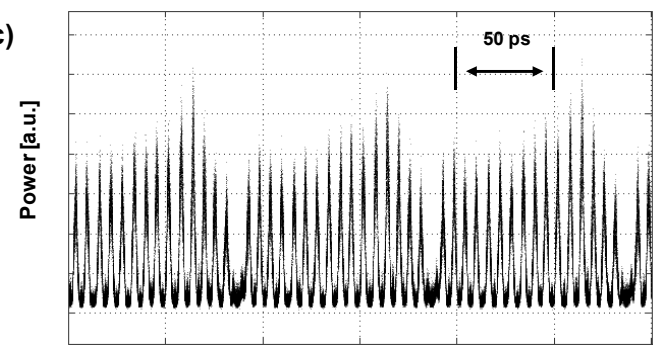

Fig. 3: Optical spectra after the first (a), second (b) FWM process in the HNLF and (c) waveform of the second idler 


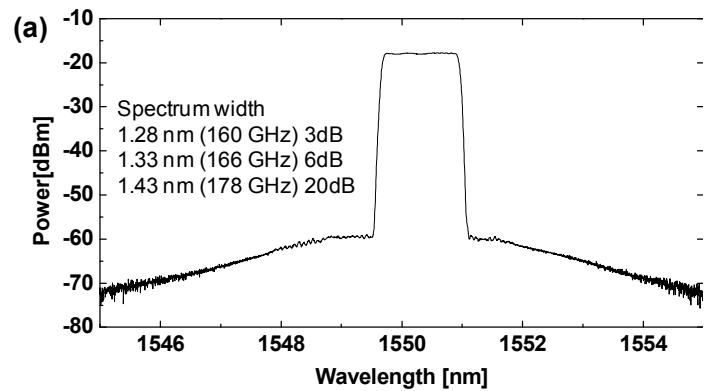

(b)

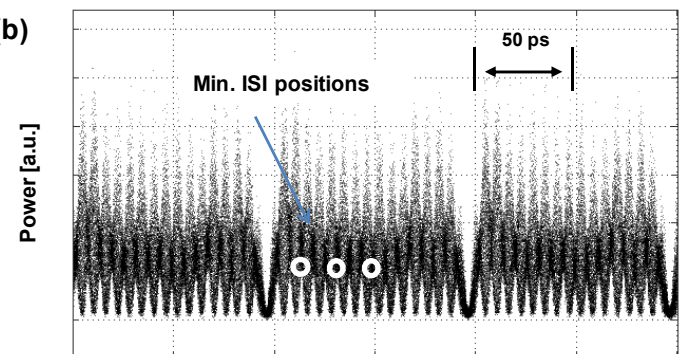

Fig. 4: Obtained Nyquist channel (a) optical spectrum, (b) temporal waveform

lower levels increases slightly (e.g. $178 \mathrm{GHz}$ at the $20 \mathrm{~dB}$ level). Fig. 4(b) shows the Nyquist OTDM waveform, where the minimum-ISI positions are clearly observed with 6 ps spacing. These minimum-ISI positions correspond to the nulls of the sinc-shaped waveforms. The minimum-ISI position obtained in each tributary time-slot is then sampled in the NOLM, using a 1.1 ps wide control pulse for gating. Finally, the BER of each tributary is measured in a 10-Gbit/s pre-amplified DPSK receiver including a delay line interferometer (DLI) and balanced photodetection.

Fig. 5 shows the BER measurements for all 16 demultiplexed Nyquist OTDM tributaries, where error-free operation (BER $<10^{-9}$ ) is achieved for all tributaries with an average receiver sensitivity

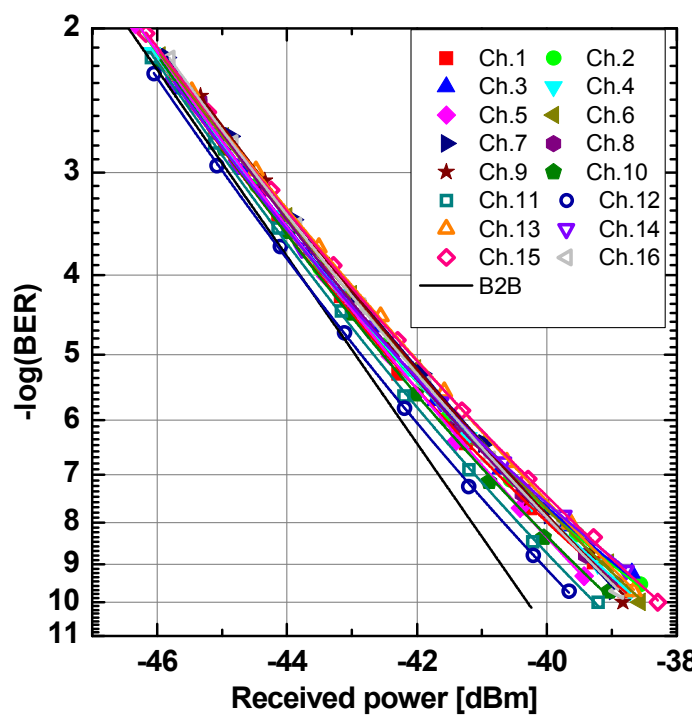

Fig. 5: BER performance of the 16 converted $10 \mathrm{Gbit} / \mathrm{s}$ DPSK Nyquist OTDM tributaries of $-39.3 \mathrm{dBm}$ at $\mathrm{BER}=10^{-9}$. For reference, the BER performance of the original DWDM channels demultiplexed with an optical tunable filter (B2B) is also plotted in Fig. 5 (only a single channel is plotted since all DWDM channels have nearly identical BER performance). The average power penalty for all 16 tributaries at $10^{-9}$ is only $1.4 \mathrm{~dB}$ for the full system. Compared to the original signal, the converted signal bandwidth is compressed from $800 \mathrm{GHz}$ to 178 $\mathrm{GHz}$ (20 dB level), resulting in a large spectral efficiency improvement from 0.2 to 0.9 symbol/s/Hz. Note that the optical sampling in the receiver could be replaced with a time-tofrequency converter (based on time-domain OFT) followed by optical bandpass filtering as in a standard DWDM receiver. Hence, our technique can potentially be made fully compatible with existing DWDM systems ${ }^{6}$.

\section{Conclusions}

We have proposed a DWDM to Nyquist channel conversion scheme based on complete OFT and optical Nyquist filtering. A $16 \times 10 \mathrm{Gbit} / \mathrm{s}$ DPSK, 50-GHz spacing DWDM signal was successfully converted to a $160 \mathrm{Gbit} / \mathrm{s}$ Nyquist channel with $178 \mathrm{GHz}$ total bandwidth, thus increasing the spectral efficiency from 0.2 to 0.9 symbol/s/Hz (theoretical limit $1 \mathrm{symbol} / \mathrm{s} / \mathrm{Hz}$ ). The average power penalty was only $1.4 \mathrm{~dB}$ for the full system. This technique may provide a simple and economical solution to upgrade commercial DWDM systems.

\section{Acknowledgment}

OFS Denmark Aps, Danish Research Council FTP project TOR (ref. no. 12-127224).

\section{References}

[1] R. J. Essiambre et al., "Capacity limits of optical fiber networks," J. Lightwave Technol., 28 (4), pp. 662-701, (2010).

[2] M. Nakazawa et al., "Ultrahigh-speed "orthogonal" TDM transmission with an optical Nyquist pulse train," Opt. Express, 20 (2), 1129-1140, (2012)

[3] M. Nakazawa et al., "Ideal distortion-free transmission using optical Fourier transformation and Fourier transform-limited optical pulses," IEEE PTL. 16, 10591061, (2004).

[4] E. Palushani et al., "OTDM-to-WDM conversion based on time-to-frequency mapping by time-domain optical Fourier transformation," IEEE J. Sel. Top. Quantum Electron. 18 (2), 681-688, (2012).

[5] P. Guan et al., "Real-Time All-Optical OFDM Transmission System Based on Time-Domain Optical Fourier Transformation," OFC'14, W4F.1, (2014).

[6] $\mathrm{H}$. Hu et al., "1.28 Tbaud Nyquist signal transmission using time-domain optical Fourier transformation based receiver," in CLEO, CTh5D.5. (2013). 
